\title{
Combined Fit of the Spectrum and Composition from Telescope Array
}

\section{Douglas R. Bergman*, for the Telescope Array Collaboration ${ }^{\dagger}$}

Dept. of Physics \& Astronomy and High Energy Astrophysics Inst., University of Utah

E-mail: bergman@physics . utah.edu

\begin{abstract}
We present the result of a fit of an astrophysical model of UHECR sources to the energy spectrum measured using the TA surface detectors and the composition data measured by TA fluorescence detectors in stereo mode. The astrophysical model consists of identical sources, uniformly distributed in comoving volume, where groups of nuclei with varying fractions are accelerated producing a power law source with a rigidity-dependent cutoff. The model includes the propagation effects of photo-pion production, photo-nuclear disintegration and general energy loss from universal expansion. Comparison of model data with detector data in both the energy and the $X_{\max }$ distributions is done via a forward-folding description of detector effects.
\end{abstract}

36th International Cosmic Ray Conference -ICRC2019-

July 24th - August 1st, 2019

Madison, WI, U.S.A.

\footnotetext{
${ }^{*}$ Speaker.

${ }^{\dagger}$ for collaboration list see PoS(ICRC2019)1177
} 


\section{Introduction and Model}

The origin of Ultra High Energy Cosmic Rays (UHECRs, $E>10^{18} \mathrm{eV}$ ) remains undetermined despite a reasonable sample of events collected by very large observatories around the world. UHECRs are believed to be of extragalactic origin, because of the observed anisotropy of arrival directions and the diffusion scale for magnetic field within the galaxy.

Using a relatively simple model of UHECR sources we use Telescope Array (TA) measurements of the energy spectrum and the observed distributions of $X_{\max }$, the depth in the atmosphere where extensive air showers reach their maximum size, to constrain possible UHECR source parameters.

The model we use is the same as that previous used by the Pierre Auger Observatory in a similar fit [1]. The source model assumes that UHECR sources are uniformly distributed (with a constant density in a co-moving volume) throughout the universe with identical properties for each source. Each source has an identical mix of five nuclei $(\mathrm{H}, \mathrm{He}, \mathrm{N}, \mathrm{Si} \mathrm{Fe})$ that are accelerated to give spectra with identical power law slopes and a rigidity-dependent exponential cutoff. The source parameters are therefore the spectral slope, the cutoff rigidity and five fractions (which sum to unity).

The model is constrained by the TA surface detector (SD) spectrum measurement (as presented at the 2017 ICRC [2]) and the TA Stereo Composition measurement (also from the 2017 ICRC [3]). We choose the TA SD spectrum because it has the largest set of statistics for a UHECR energy spectrum measured in the Northern Hemisphere. It is also different from the spectrum measured in the Southern Hemisphere with a higher energy for the final sharp break in the spectrum ( $60 \mathrm{EeV} c f$. $40 \mathrm{EeV}$ ). We choose the TA Stereo composition measurement because of its indifference to high energy particle interaction models.

The effects of propagation of nuclear species through the universe is done by the CRPropa model [4]. The CRPropa calculation was done using both the cosmic microwave background (CMB) and the Gilmore model [5] of the infrared background (IRB) to calculate photo-pion production, photo-nuclear disintegration and electron-positron pair-production. The cosmological parameters used were those determined by Planck in 2015 [6]. A 1-D propagation calculation was performed for a series of small ranges in $z$, "shells", with 400 shells logarithmically spaced between $z=10^{-4}$. 1 million particles of each source species were injected with an $E^{-1}$ spectrum between the energies of 0.0316 and $3160 \mathrm{EeV}$. Each "observed" nuclei was recorded and binned by atomic number. Thus for each input species, each possible output species and each shell, we create a $500 \times 500$ bin $E_{\text {out }} / E_{\text {in }}$ histogram. ( $E_{\text {in }}$ is the injected energy, $E_{\text {out }}$ is the energy at observation) These can be combined into big arrays, e.g., for Fe we have a $56 \times 400 \times 500 \times 500$ array. As this gets to be an unruly size of data, we sum over the $400 z$-shell bins weighted by the light-travel time across the shell (i.e. the thickness of the shell) and the density of sources in the shell (given by the co-moving constant density assumption $\left.(1+z)^{3}\right)$. Using the $E_{\text {out }} / E_{\text {in }}$ histograms then we can put in any input (source) spectrum and get the expected "observed" spectrum of a particular nuclear type. The input spectrum is created according to the model given above.

For comparison with the spectrum we use this output directly. For comparison with the stereo composition measurement we use the Gumbel [7] distribution to create $X_{\max }$ distributions for each nuclear type and energy. The parameters for the Gumbel distribution for given high-energy interac- 
tion models has been tabulated in [7]. We must then also apply a stereo $X_{\max }$ acceptance weighting to these distributions and smear according to the stereo $X_{\max }$ resolution. The $X_{\max }$ acceptance was calculated in the energy bins used and then fit to a model of a flat-top plateau, a breakpoint in $X_{\max }$ and an exponential fall-off in acceptance above the break point. The $X_{\max }$ acceptance is shown in Figure 1. The stereo $X_{\max }$ resolution was calculated by comparing the generated and the reconstructed $X_{\max }$ values for simulated proton and iron primaries using in each energy band. This determination of the $X_{\max }$ reoslution is the same as that determined from the stereo composition analysis [3]. The values for the $X_{\max }$ resolution used in this analysis are stated in Table 1. The $X_{\max }$ distribution resulting from the output of the Gumbel model and with the acceptance model applied, was then smeared using a single gaussian in $X_{\max }$ with a width given by logarithmic interpolation between the tabulated proton and iron resolutions based on the known primary nuclear mass. The sum of these $X_{\max }$ distributions for all the observed primary cosmic ray species was then compared to the observed $X_{\max }$ distribution.

For both the spectrum and composition analyses we use a binned log-likelihood comparison of events observed compared to events in the model. For the spectrum comparison the log-likelihood is based on the Poisson distributions, while for the $X_{\max }$ comparison, the log-likelihood is based on a multinomial distribution. Both log-likelihood comparisons are scaled to produce a $\chi^{2}$-like distribution in the limit of very large numbers of events. We refer to these as deviances, and sum the deviance from the spectrum and the deviance from the compositions analysis to determine an overall figure-of-merit for the model with given parameters.
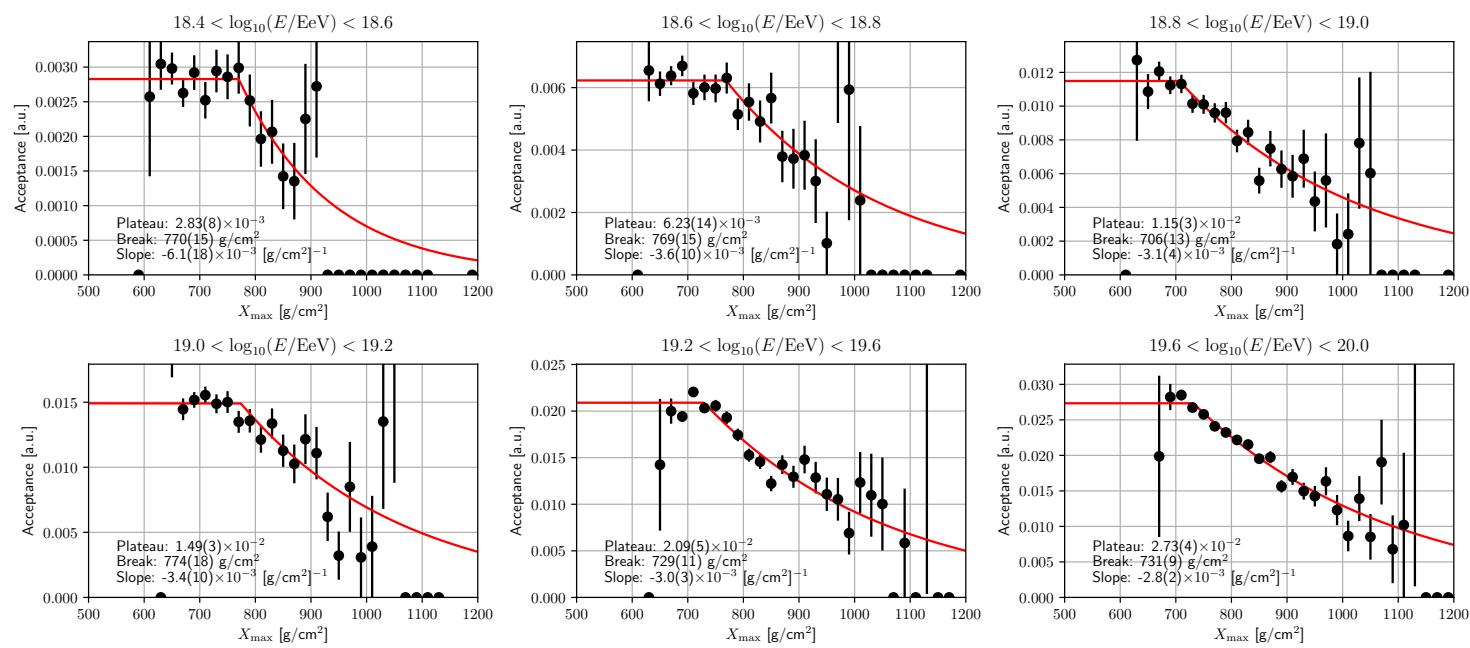

Figure 1: Stereo $X_{\max }$ acceptance calculated from QGSJetII-04 simulations of iron and proton primaries. The acceptance is fit to a model with a plateau at small $X_{\max }$, a break point in $X_{\max }$, and an exponential decrease in acceptance with $X_{\max }$ above the break point. The fits were performed using a binned log-likelihood minimization.

\section{Combined Fit Results}

For our baseline fit result we used the QGSJetII-04 high-energy interaction model and the 


\begin{tabular}{|l|c|c|c|c|c|c|}
\hline \hline Energy Range & $18.4-18.6$ & $18.6-18.8$ & $18.8-19.0$ & $19.0-19.2$ & $19.2-19.6$ & $19.6-20.0$ \\
\hline proton & 21.8 & 19.0 & 17.6 & 16.3 & 16.4 & 16.4 \\
Iron & 17.8 & 16.8 & 14.8 & 13.2 & 13.0 & 12.8 \\
\hline \hline
\end{tabular}

Table 1: Table of $X_{\max }$ resolutions in $\mathrm{g} / \mathrm{cm}^{2}$. Energy ranges are given in $\log _{10}(E / \mathrm{EeV})$

Gilmore-12 IRB model. We scanned the $\gamma-\log _{10}\left(R_{\max } / \mathrm{EV}\right)$ plane, and for each pair allowed the five source fractions to vary under the requirement that the fractions sum to unity. $\gamma$ was scanned in 401 steps from $-1.5-2.5$ while $\log _{10} R$ was scanned in 401 steps from $-0.5-3.5$. The deviance contours corresponding to $10-\sigma$ are shown on the left in Figure 2. The best fit point is at $(2.00,3.18)$ with a total deviance $D=157.5$ which we will call Scenario A. There is a local minimum on the left edge of the plot at the point $(-1.50,0.35)$ with $D=184.8$ which we will call Scenario B.

As an alternative to the above fit, we also used the EPOS-LHC high-energy interaction model with the Gilmore-12IRB model, and shifted the TA data down in energy by $10.2 \%$ to match the energy scale difference between Auger and TA found by the TA/Auger Spectrum Working Group. The scan was done over the same range and with the same fineness, and the result is shown on the right in Figure 2. The best fit point is at $(1.95,3.14)$ with a total deviance $D=195.6$ which we will call Scenario C. There is a local minimum at the point $(0.79,0.74)$ with $D=226.0$ which we will call Scenario D. The deviances for both Scenario C and D are greater than those for either Scenario A or B.

The details of the fit for Scenario A $\left(\gamma=2.00, R_{\max }=1514 \mathrm{EV}\right)$ are shown in Figures 3 and 4. The source fractions are: $0.0 \%, 56.3 \%, 31.0 \%, 7.5 \%$, and $5.1 \%$ for $\mathrm{H}, \mathrm{He}, \mathrm{N}, \mathrm{Si}$, and $\mathrm{Fe}$, respectively. The fit is dominated by the very large flux in TA above $10^{1.7} \mathrm{EeV}$ which is above the model's prediction for a GZK-effect.

The details of the fit for Scenario B $\left(\gamma=-1.50, R_{\max }=2.239 \mathrm{EV}\right)$ are shown in Figures 5 and 6. The source fractions are: $61.0 \%, 37.4 \%, 1.5 \%, 0.1 \%$, and $0.003 \%$ for $\mathrm{H}, \mathrm{He}, \mathrm{N}, \mathrm{Si}$, and $\mathrm{Fe}$, respectively.

Scenarios C and D offer a more direct comparison to the Auger Combined Fit with the shift of the TA energies and the use of the same high-energy interaction model. Scenario $\mathrm{C}$ is quite similar to Scenario A and we will not show the fit results here. It also has no $\mathrm{H}$ at the source.

The details of the fit for Scenario D $\left(\gamma=0.79, R_{\max }=5.495 \mathrm{EV}\right)$ are shown in Figures 7 and 8. The source fractions are: $61.0 \%, 37.4 \%, 1.5 \%, 0.1 \%$, and $0.003 \%$ for $\mathrm{H}, \mathrm{He}, \mathrm{N}, \mathrm{Si}$, and $\mathrm{Fe}$, respectively.

\section{Discussion}

From the several fit results shown of the Telescope Array spectrum and stereo composition data, a number of different interpretations of source compositions could be drawn. Not all of these source compositions are astrophysically plausible, e.g., predominantly $\mathrm{He}$ with no $\mathrm{H}$; or predominantly $\mathrm{H}$ with no He nor $\mathrm{N}$ but significant $\mathrm{Si}$ and a little Fe. The best fit for the TA data overall shows that one might expect predominantly $\mathrm{H}$ at observation although no $\mathrm{H}$ was accelerated 

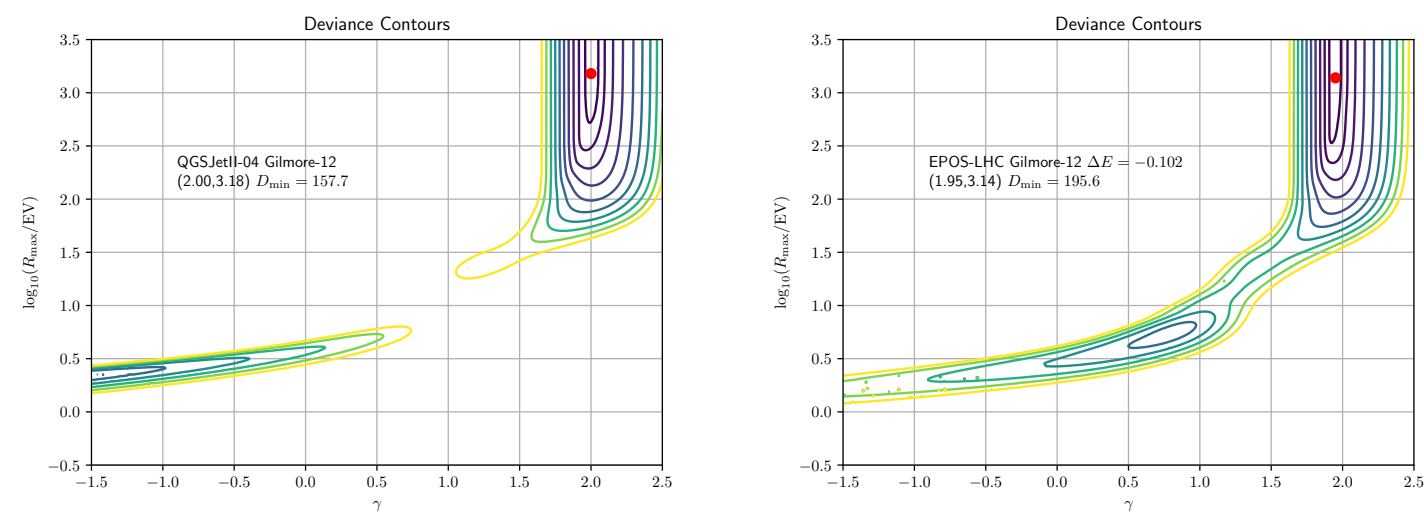

Figure 2: Left: A plot of the deviance contours corresponding to 10- $\sigma$ for Telescope Array data using QGSJetII-04 as the high-energy interaction model and the Gilmore-12 IRB model. The best fit point is $\gamma=2.00, \log _{10}\left(R_{\max } / \mathrm{EV}\right)=3.18\left(R_{\max }=1514 \mathrm{EV}\right)$. This point had source fractions: $0.0 \%, 56.3 \%, 31.0 \%$, $7.5 \%$, and $5.1 \%$ for $\mathrm{H}, \mathrm{He}, \mathrm{N}, \mathrm{Si}$, and Fe, respectively. The local minimum at $\gamma=-1.5, \log _{10}\left(R_{\max } / \mathrm{EV}\right)=$ $0.35\left(R_{\max }=2.239 \mathrm{EV}\right)$ has source fractions: $61.0 \%, 37.4 \%, 1.5 \%, 0.1 \%$, and $0.003 \%$ for $\mathrm{H}, \mathrm{He}, \mathrm{N}, \mathrm{Si}$, and Fe, respectively. Right: A plot of the deviance contours corresponding to 10- $\sigma$ for energy shifted Telescope Array data (by -10.2\%) using EPOS-LHC as the high-energy interaction model and the Gilmore12 IRB model. The best fit point is $\gamma=1.95, \log _{10}\left(R_{\max } / \mathrm{EV}\right)=3.14\left(R_{\max }=1380 \mathrm{EV}\right)$. This point had source fractions: $0.0 \%, 24.0 \%, 56.6 \%, 0.007 \%$, and $19.4 \%$ for $\mathrm{H}, \mathrm{He}, \mathrm{N}, \mathrm{Si}$, and $\mathrm{Fe}$, respectively. The local minimum at $\gamma=0.79, \log _{10}\left(R_{\max } / \mathrm{EV}\right)=0.74\left(R_{\max }=5.495 \mathrm{EV}\right)$ has source fractions: $80.1 \%, 0 \%, 0 \%$, $17.5 \%$, and $2.4 \%$ for $\mathrm{H}, \mathrm{He}, \mathrm{N}, \mathrm{Si}$, and $\mathrm{Fe}$, respectively.
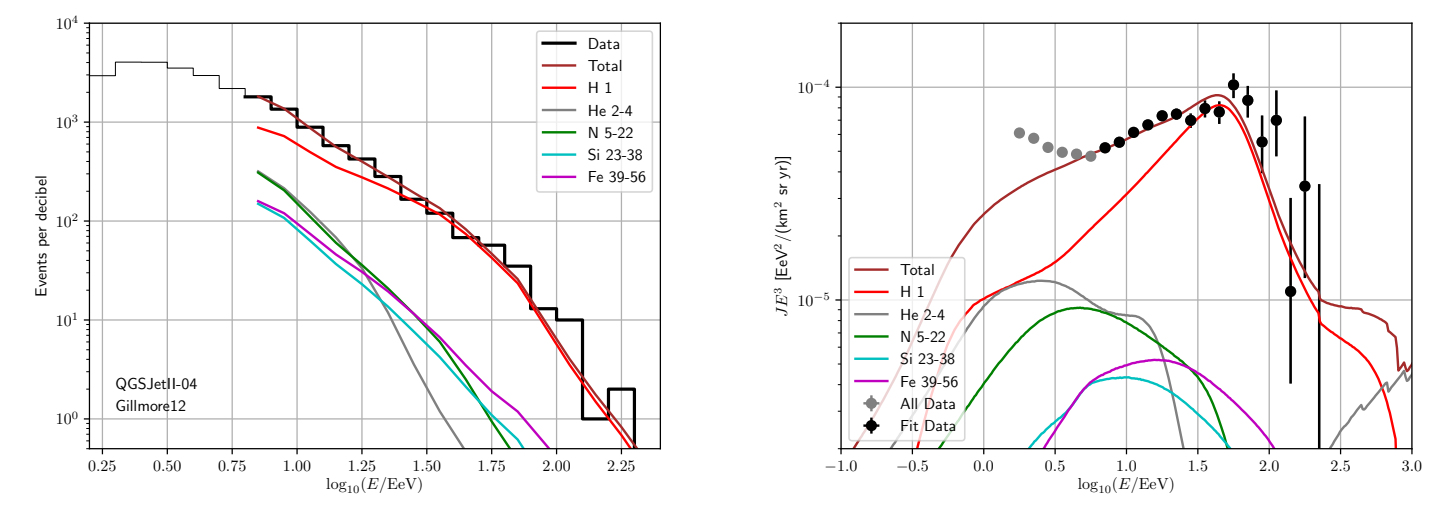

Figure 3: Left: The observed event energy distribution and the model predictions for observed species in Scenario A. The best fit gives a predominantly $\mathrm{H}$ at observation, despite no $\mathrm{H}$ at the source. Right: The observed and predicted energy flux spectra (multiplied by $E^{3}$ ) for Scenario A. 

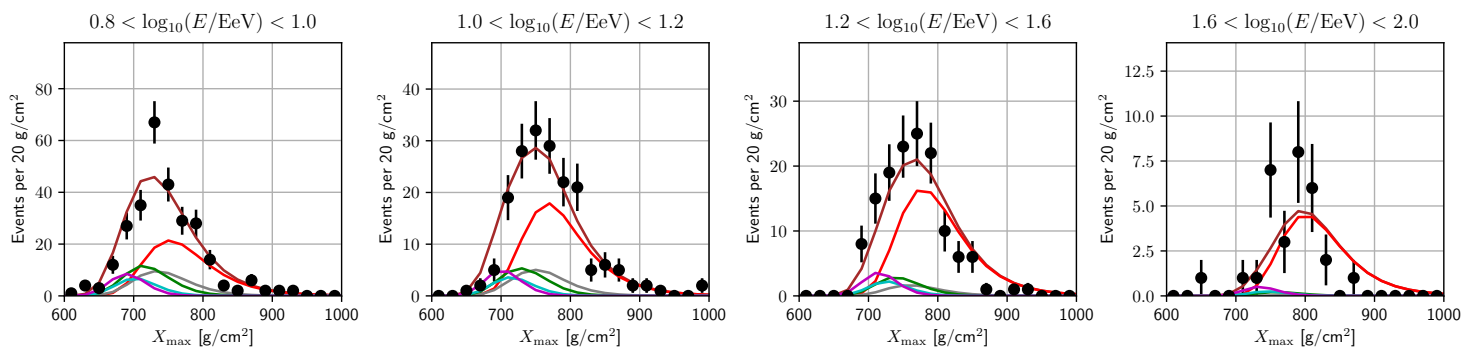

Figure 4: The observed $X_{\max }$ distributions in bins of energy along with the predicted $X_{\max }$ distributions from the model in Scenario A. The colors of the observed elemental group predictions is the same as in Figure 3.
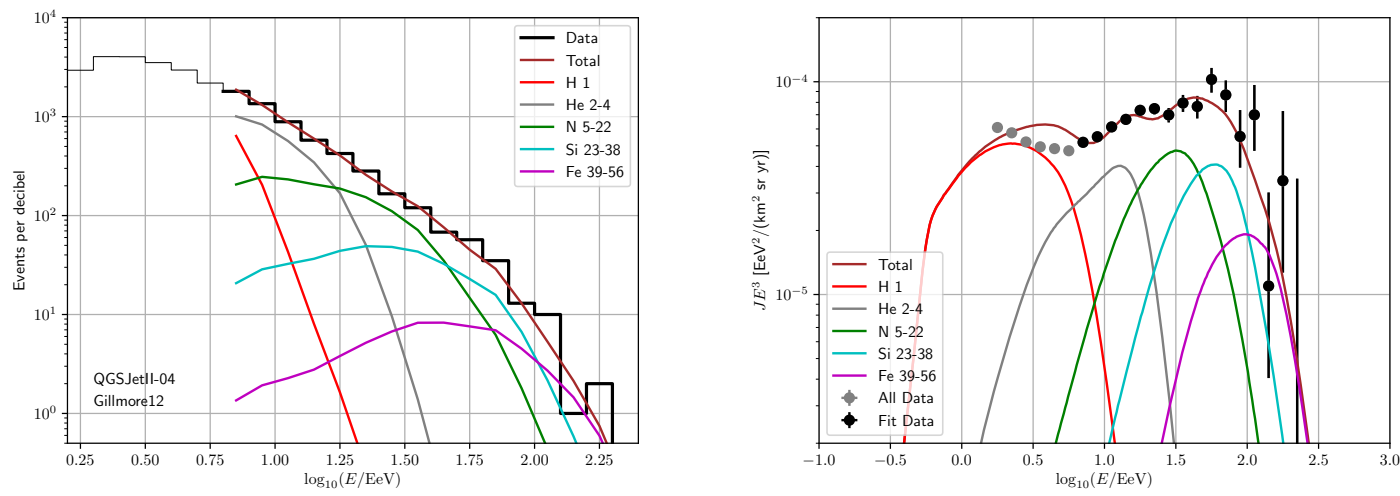

Figure 5: Left: The observed event energy distribution and the model predictions for observed species in Scenario B. Right: The observed and predicted energy flux spectra (multiplied by $E^{3}$ ) for Scenario B.
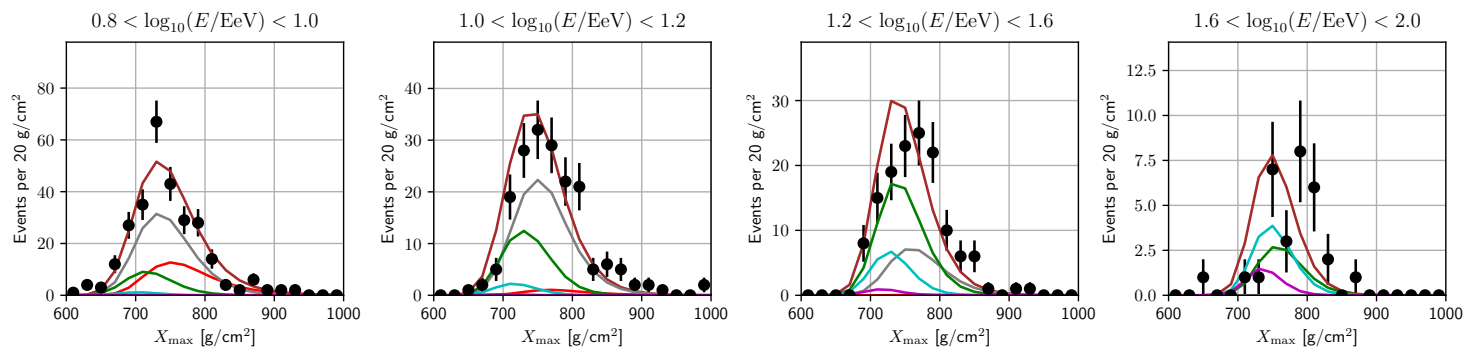

Figure 6: The observed $X_{\max }$ distributions in bins of energy along with the predicted $X_{\max }$ distributions from the model in Scenario B. The colors of the observed elemental group predictions is the same as in Figure 3. 

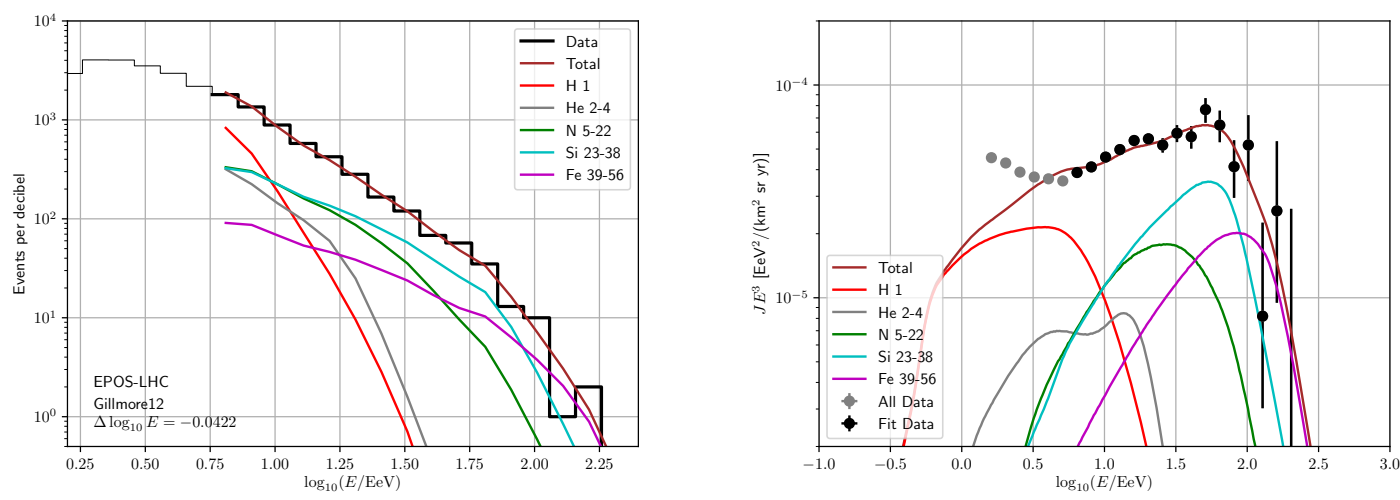

Figure 7: Left: The observed event energy distribution and the model predictions for observed species in Scenario D. Right: The observed and predicted energy flux spectra (multiplied by $E^{3}$ ) for Scenario D.
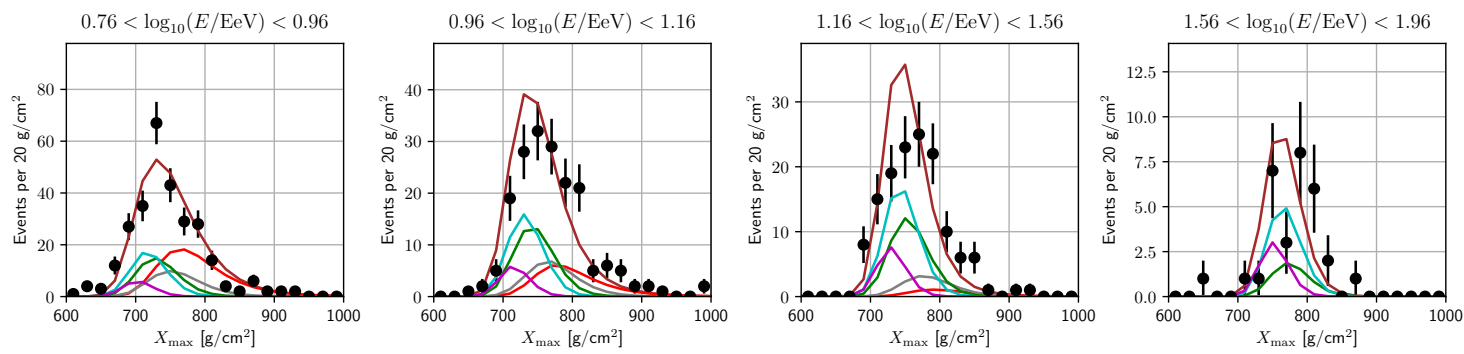

Figure 8: The observed $X_{\max }$ distributions in bins of energy along with the predicted $X_{\max }$ distributions from the model in Scenario D. The colors of the observed elemental group predictions is the same as in Figure 3.

at the source. This is quite different from the interpretation gleaned from the combined fit to the Auger data, which showed a moderately hard spectrum at the source contributing to a progressively heavier set of dominant species at observation. Since the Auger and TA spectrum data are consistent in this range except for the very highest energies, one is tempted to conclude that this combinedfit model of astrophysical sources shows an excessive sensitivity to statistical fluctuations in the energy and $X_{\max }$ distributions.

\section{Acknowledgments}

The Telescope Array experiment is supported by the Japan Society for the Promotion of Science(JSPS) through Grants-in-Aid for Priority Area 431, for Specially Promoted Research JP21000002, for Scientific Research (S) JP19104006, for Specially Promoted Research JP15H05693, for Scientific Research (S) JP15H05741, for Science Research (A) JP18H03705 and for Young Scientists (A) JPH26707011; by the joint research program of the Institute for Cosmic Ray Research (ICRR), The University of Tokyo; by the U.S. National Science Foundation awards PHY-0601915, PHY-1404495, PHY-1404502, and PHY-1607727; by the National Research Foundation of Korea 
(2016R1A2B4014967, 2016R1A5A1013277, 2017K1A4A3015188, 2017R1A2A1A05071429) ; by the Russian Academy of Sciences, RFBR grant 16-02-00962a (INR), IISN project No. 4.4502.13, and Belgian Science Policy under IUAP VII/37 (ULB). The foundations of Dr. Ezekiel R. and Edna Wattis Dumke, Willard L. Eccles, and George S. and Dolores Doré Eccles all helped with generous donations. The State of Utah supported the project through its Economic Development Board, and the University of Utah through the Office of the Vice President for Research. The experimental site became available through the cooperation of the Utah School and Institutional Trust Lands Administration (SITLA), U.S. Bureau of Land Management (BLM), and the U.S. Air Force. We appreciate the assistance of the State of Utah and Fillmore offices of the BLM in crafting the Plan of Development for the site. Patrick Shea assisted the collaboration with valuable advice on a variety of topics. The people and the officials of Millard County, Utah have been a source of steadfast and warm support for our work which we greatly appreciate. We are indebted to the Millard County Road Department for their efforts to maintain and clear the roads which get us to our sites. We gratefully acknowledge the contribution from the technical staffs of our home institutions. An allocation of computer time from the Center for High Performance Computing at the University of Utah is gratefully acknowledged.

\section{References}

[1] The Pierre Auger collaboration, Combined fit of spectrum and composition data as measured by the Pierre Auger Observatory, JCAP(2017)038. [arXiv:1612.07155]

[2] Y. Tsunesada et al., Energy Spectrum of Ultra-High-Energy Cosmic Rays Measured by The Telescope Array, PoS(ICRC2017)535.

[3] T. Stroman, D. Bergman, Telescope Array measurement of UHECR composition from stereoscopic fluorescence detection, $\mathrm{PoS}(\mathrm{ICRC}) 538$.

[4] R.A. Batista et al., CRPropa 3 - a Public Astrophysical Simulation Framework for Propagating Extraterrestrial Ultra-High Energy Particles, JCAP05(2016)038. [arXiv:1603.07142]

[5] R.C. Gilmore et al., Semi-analytic modeling of the EBL and consequences for extragalactic gamma-ray spectra, Mon. Not. Roy. Astron. Soc. 422 (2012) 3189. [arXiv:1104.0671]

[6] Planck Collaboration, Planck 2015 results. XIII. Cosmological parameters, A\&A 594, A13 (2016) [arXiv:1502.01589]

[7] M. De Dominico et al., Reinterpreting the development of extensive air showers initiated by nuclei and photons, JCAP07(2013)050. [arXiv:1305.2331] 